\title{
Optimization of Land Surface Parameters for Weather Simulations over Arid and Semi-Arid Regions
}

\author{
WoonSeon Jung ${ }^{1}$, Masataka Murakami ${ }^{1,2}$, Taro Shinoda ${ }^{1}$, and Masaya Kato ${ }^{1}$ \\ ${ }^{1}$ Institute for Space-Earth Environmental Research, Nagoya University, Nagoya, Japan \\ ${ }^{2}$ Meteorological Research Institute, Japan Meteorological Agency, Tsukuba, Japan
}

\begin{abstract}
The Cloud Resolving Storm Simulator (CReSS) model with default parameter settings largely underestimates the diurnal variation of land surface temperature (LST) and hence the formation of summertime diurnal convective clouds over the United Arab Emirates (UAE), which are the primary target of cloud seeding in the UAE. Based on sensitivity experiment results, we optimized a set of land surface parameters to minimize the underestimation of LST and improve the performance of weather simulations over deserts and mountains in the UAE. In the optimized experiment, the difference in LST between Aqua/MODIS observations and CReSS simulation results decreased from 13.0 to $2.3 \mathrm{~K}$ under daytime conditions, mainly due to decreased evapotranspiration efficiency and soil thermal diffusivity. Moreover, the difference decreased from 3.8 to $2.0 \mathrm{~K}$ under nighttime conditions, mainly due to decreased evapotranspiration efficiency and increased soil temperature at the deepest layer. A decrease in roughness length suppressed the increase in surface air temperature (SAT), contributing to the reproduction of a realistically large difference between LST and SAT during the daytime. The improvement in temperature matching demonstrates that the CReSS model, when used at a $1-\mathrm{km}$ horizontal resolution, accurately simulates the formation of diurnal convective clouds and development of precipitation over deserts in the UAE and low mountains in northeastern UAE.
\end{abstract}

(Citation: Jung, W., M. Murakami, T. Shinoda, and M. Kato, 2018: Optimization of land surface parameters for weather simulations over arid and semi-arid regions. SOLA, 14, 197-202, doi: 10.2151/sola.2018-035.)

\section{Introduction}

Approximately one-third of the globe can be described as arid $(12.1 \%)$ or semi-arid (17.7\%) (Warner 2009), meaning that such areas receive less than 200 or $500 \mathrm{~mm}$ of precipitation per year, respectively. The United Arab Emirates (UAE) is surrounded by several deserts, such as the Al Khatim Desert, Arabian Desert, Gulf of Oman desert and semi-desert, and Rub al-Khali. The climatology from 1991 to 2015 over the UAE, the World Bank's publication (http://sdwebx.worldbank.org/climateportal/index.cfm?page= downscaled data download\&menu=historical), showed that rainfall was concentrated during November to April and the dry season occurred from May to October. The average annual precipitation during that period was $57 \mathrm{~mm}$, indicating an arid climate. According to the land-cover classification provided by the United States Geological Survey, most land types in the UAE and surrounding areas are shrubland (LT1) and barren or sparsely vegetated land (LT2).

To conduct forecast and hindcast simulations for use in rain enhancement research over the UAE, accurate and reliable numerical models are needed to simulate cloud formation and precipitation development over arid and semi-arid regions. The main target

Corresponding author: WoonSeon Jung, Institute for Space-Earth Environmental Research, Nagoya University, Furo-cho, Chikusa-ku, Nagoya, Aichi 464-8601, Japan. E-mail: wsjung@rain.isee.nagoya-u.ac.jp.

*Present affiliation: National Institute of Meteorological Sciences, 33, Seohobuk-ro, Seogwipo-si, Jeju-do, 63568, Republic of Korea. E-mail: wsjung01@korea.kr. of cloud seeding for rain enhancement in the UAE is thought to be summertime diurnal convective clouds, which form through the development of a convective boundary layer. Surface energy and water budgets for deserts are key constraints in the development of this layer. In particular, land surface temperature (LST) is well known as the key parameter in the physical processes of surface energy and water budgets at local through global scales ( $\mathrm{Li}$ et al. 2013). Hashimoto et al. (2017) optimized the land surface parameters over the UAE used in the Meteorological Research Institute non-hydrostatic model. However, the parameterization of land surface processes in their model differs considerably from ours, and they validated their model against observations in terms of seasonal and regional distributions of precipitation. Therefore, the purpose of the numerical modeling study described in this paper is to optimize a set of land surface parameters for desert areas for use in the Cloud Resolving Storm Simulator (CReSS; Tsuboki and Sakakibara 2007) model and to validate and improve this numerical model for weather simulations, especially regarding the formation of diurnal convective cloud and precipitation development over the UAE.

\section{Numerical model and experimental design}

Non-hydrostatic cloud-resolving models, of which the CReSS model is a sophisticated and popular example, are used to numerically simulate cloud formation and precipitation development. The CReSS model has mainly been used for the simulation of wet weather, such as typhoons, heavy rainfall, and snowfall, in lowand mid-latitudes and has shown good reproducibility of these phenomena (Ohigashi and Tsuboki 2007; Yamada et al. 2007; Shinoda et al. 2009; Lee et al. 2010; Hirata et al. 2016; Wang et al. 2016; Kanada et al. 2017). However, there are no published reports of the CReSS model having been used to simulate dry weather over desert areas.

The CReSS model comprises the following components: bulk cloud microphysical parameterization of cold rain processes, with a single-moment scheme for liquid hydrometeors and a double-moment scheme for solid hydrometeors (Murakami et al. 1994); turbulence processes formulated with 1.5-order turbulence kinetic energy (Deardoff 1980); and surface processes described using an aerodynamic bulk formula (Kondo 1976). The values of land surface parameters are based on those in the fifth-generation Pennsylvania State University-National Center for Atmospheric Research Mesoscale Model known as MM5 (Dudhia et al. 2004).

Numerical simulations with a $5-\mathrm{km}$ horizontal resolution (CReSS_5km) were conducted for 9-11 September 2015, during which typical diurnal variations in LST and surface air temperature (SAT; air temperature at $1.5 \mathrm{~m}$ above the ground) were observed that led to the formation of typical diurnal convective clouds and the development of precipitation over the mountains in Oman, deserts in the UAE, and low mountains in northeastern UAE, using the Japan Meteorological Agency's Global Spectral Model forecast as the initial and boundary data. Numerical simulations were also performed for typical clear day cases in the cold (15-17 February 2015) and warm (14-16 August 2015) seasons. In addition, numerical simulations with a $1-\mathrm{km}$ horizontal resolution (CReSS_1 km, which was one-way nested in CReSS_5km) were performed to examine the reproducibility of cloud forma- 
tion and precipitation development in as accurate and detailed a manner as possible. Figure 1 shows the model domains of CReSS $5 \mathrm{~km}$ and CReSS $1 \mathrm{~km}$, and Table S1 presents the details of the model configuration.

This paper presents the simulation results of land surface parameter sensitivity, optimization, and validation experiments using the CReSS model, mainly for 9-11 September 2015.

\section{Results and discussion}

\subsection{Sensitivity experiments}

CReSS simulations using the original (default) values of land surface parameters could not reliably reproduce the amplitude of the diurnal variation in LST and the large difference between daytime LST and SAT specific to deserts (Fig. S1). To optimize a set of land surface parameters suitable to the desert in the study area, we first investigated the major parameters influencing LST and SAT by performing sensitivity experiments for eight parameters (thermal diffusivity, thermal capacity, evapotranspiration efficiency, roughness length, soil temperature in the deepest layer, land surface emissivity, number of soil layers, and thickness of each soil layer). Then, we compared the CReSS results with $0.05^{\circ} \times 0.05^{\circ}$ spatial resolution Aqua/MODIS data for LST and data from 45 automatic weather stations (AWSs) from the UAE National Center of Meteorology for SAT and relative humidity. Figure 1 presents the model domains and land-cover types, which

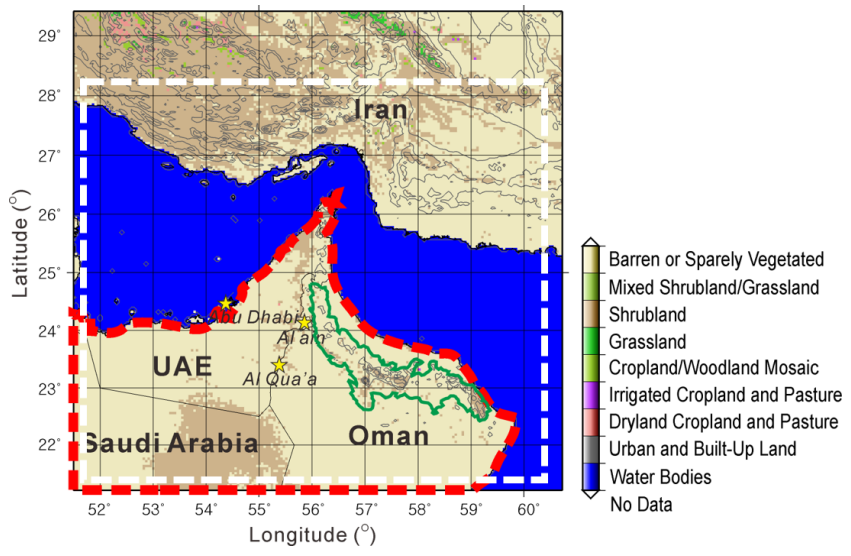

Fig. 1. Model domain of CReSS $5 \mathrm{~km}$ and land-cover types over the United Arab Emirates (UAE) and surrounding areas. The white dashed box indicates the domain of CReSS_1 $1 \mathrm{~km}$. The topography is delineated by contour lines at $500 \mathrm{~m}$ intervals. The area encircled by green line indicates the Omani mountains. The red dashed line indicates the analysis area of domain-averaged land surface temperature. Two automatic weather station sites, Al Ain (300 m MSL) and Al Qua'a (130 m MSL) were chosen as typical observation sites in the desert area for comparison with the simulation results. mainly comprise shrubland (16.2\%) and barren or sparsely vegetated land $(83.7 \%)$. LST was averaged over the analysis area, and SAT was averaged over the whole UAE.

In one group of experiments, the first four parameters (thermal diffusivity, thermal capacity, evapotranspiration efficiency, and roughness length) were each set to one-tenth of the default values, keeping all other parameters at the default values. Similarly, the last four parameters (soil temperature in the deepest layer, emissivity at the surface, number of soil layers, and thickness of each soil layer) were set to $293.16 \mathrm{~K}, 0.9,20$, and $0.01 \mathrm{~m}$, respectively. In a second group of experiments, the first four parameters were set to 10 times the default values, and the last four parameters were set to $313.16 \mathrm{~K}, 1.0,73$, and $0.10 \mathrm{~m}$, respectively. We performed $16(=2 \times 8)$ sensitivity experiments in total. The default values of the eight parameters are shown in Table 1.

Figures $2 \mathrm{a}$ and $2 \mathrm{~b}$ present the differences in LST and SAT between experiments 1 and 2 . The sensitivity experiments showed that the variations in LST during the daytime were primarily caused by changes in evapotranspiration efficiency, thermal capacity, and thermal diffusivity. SAT was largely influenced by

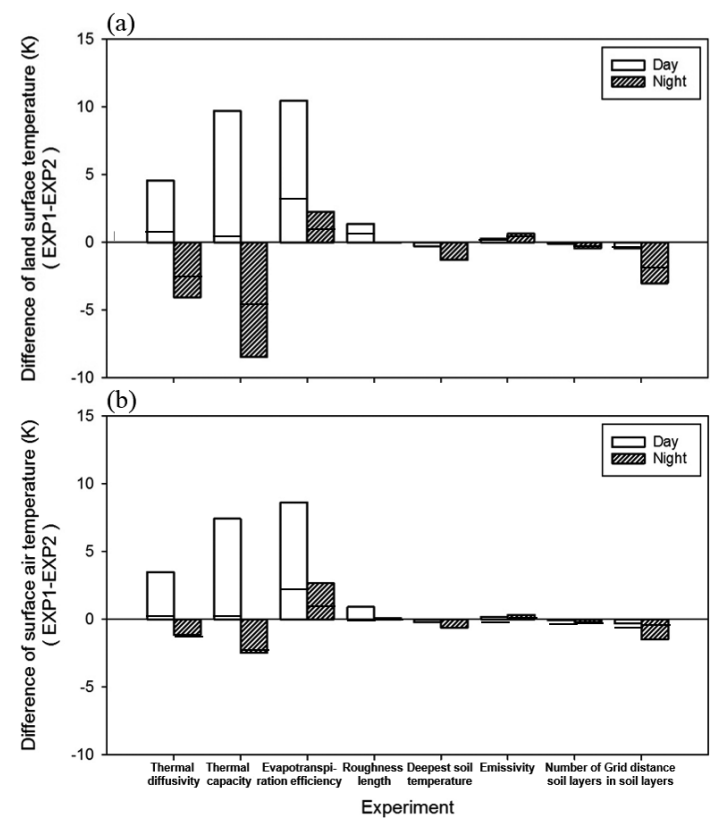

Fig. 2. Difference in (a) land surface temperature and (b) surface air temperature between experiments 1 (EXP1) (one-tenth of the default parameter value (CTL) coefficient, except for the last four parameters) and 2 (EXP2) (10 times the CTL coefficients, except for the last four parameters). The white and shaded bars denote the values during the day (14:00 local time on 10 September 2015) and at night (02:00 local time on 11 September 2015). The horizontal line in each bar indicates the difference between EXP1 and CTL.

Table 1. The values of land surface parameters for control and optimization experiments. The number of soil layers and thickness of soil layers are common to all land-cover types shown in Fig. 1.

\begin{tabular}{lcl}
\hline & CTL experiment & \multicolumn{1}{c}{ OPT experiment } \\
\hline Thermal diffusivity $\left[\mathrm{m}^{2} \mathrm{~s}^{-1}\right]$ & $5 . \mathrm{e}-7 / 5 . \mathrm{e}-7$ & $5.0 \mathrm{e}-7 / 2.4 \mathrm{e}-7$ (List, 1966) \\
Thermal capacity $\left[\mathrm{J} \mathrm{m}^{-3} \mathrm{~K}^{-1}\right]$ & $1.77 \mathrm{e} 6 / 1.18 \mathrm{e} 6$ & $1.77 \mathrm{e} 6 / 1.28 \mathrm{e} 6$ (List, 1966) \\
Evapotranspiration efficiency & $0.10 / 0.02$ & $0.10 / 0.01$ \\
Roughness length of momentum $[\mathrm{m}]$ & $0.05 / 0.01$ & 0.0019 (Zhang et al., 2003) $)^{(\mathrm{b})}$ \\
Roughness length of heat and water vapor $[\mathrm{m}]$ & 0.1 & 0.00043 (Zhang et al., 2003) \\
Soil temperature in deepest layer $[\mathrm{K}]$ & 288.16 & 310.15 (Takano et al., 1999) $^{(\mathrm{c})}$ \\
Surface Emissivity & 0.95 & 0.90 (Berge, 1990) $^{(\mathrm{d})}$ \\
Number of soil and sea layers & 43 & 30 \\
Thickness of soil layers $[\mathrm{m}]$ & 0.05 & 0.02 \\
\hline
\end{tabular}

(a) Sandy soil material, (b) Observation in Gobi Desert, (c) Observation in UAE, (d) Dry sand (LT1: Shrubland/LT2: Barren or sparely vegetated) 
LST, and the equivalent daytime changes were slightly smaller than those of LST. At night, thermal capacity, evapotranspiration efficiency, thermal diffusivity, and the thickness of soil layers had major influences on LST and SAT. In Fig. 2, positive values in the changes of LST and SAT indicate that a decrease in parameters caused an increase in temperature. The changes in LST and SAT caused by the changes in the parameters around the default values were not necessarily symmetrical. Moreover, the sensitivities of LST and SAT to the changes in parameters around the default values were underestimated, because the set of default parameters led to a large thermal inertia in the reactor (i.e., land surface system) compared with the optimized parameters. In addition, the sensitivities of LST and SAT depended on the values of other parameters to some extent. The magnitude of the temperature increase due to the optimization of each parameter therefore also depended on the order in which the parameters were optimized.

\subsection{Optimization experiments}

On the basis of the sensitivity experiment results, numerical experiments were performed to optimize the parameters using a trial-and-error method. After narrowing the range of the parameters, we adopted values for desert areas reported in the literature that were within the value ranges. Table 1 shows the default (CTL) and optimized (OPT) parameter values. Thermal diffusivity and capacity were taken from the properties of sandy soil material reported by List (1966). The parameters of roughness length for momentum, heat, and water vapor were derived from observations from the Gobi Desert reported by Zhang et al. (2003). Soil temperature in the deepest layer in the CReSS model was set to the observed value at a depth of $1 \mathrm{~m}$ at a study site in Ras al-Khaimah, UAE, as reported by Takano et al. (1999). Lastly, land surface emissivity was based on the properties of dry sand reported by Berge (1990).

Considering the magnitude and direction (i.e., increase/ decrease) of the change in each parameter for the optimization, the most influential change causing an increase in LST during the daytime was a decrease in evapotranspiration efficiency, followed by decreases in thermal diffusivity of soil and roughness length. For the optimization, the changes in soil heat capacity, number of soil layers, and thickness of soil layers could not cause an increase in LST. Meanwhile, the primary changes resulting in an increased LST at night were a decrease in evapotranspiration efficiency and an increase in soil temperature at the deepest layer, followed by a decrease in land surface emissivity and an increase in soil heat capacity. To increase the difference between LST and SAT (i.e., suppress the overestimation of SAT) during the daytime, roughness length for momentum and heat/water vapor were also optimized.

Figure 3 shows the horizontal distributions and histograms of LST during the day- and nighttime obtained by Aqua/MODIS observations and simulations using CReSS CTL $5 \mathrm{~km}$ and CReSS_OPT $5 \mathrm{~km}$. The results of CReSS_CTL $\overline{5} \mathrm{~km}$ and CReSS OPT_5 $\overline{\mathrm{k}} \mathrm{m}$ showed similar horizontal patterns to the Aqua/MODIS observations for both day- and nighttime. However, the difference between the domain-averaged LSTs from MODIS observations and CReSS simulations decreased from $13.0 \mathrm{~K}$ in the CTL to 2.3 $\mathrm{K}$ in the OPT experiments in the daytime. The difference at night decreased from $3.8 \mathrm{~K}$ in the CTL to $2.0 \mathrm{~K}$ in the OPT experiments. Note that the domain-averaged LST from the CReSS simulations was calculated excluding areas lacking MODIS data. The histogram of LST simulated by CReSS_OPT_5km showed better agreement with that observed by MODIS during both the day- and nighttime.

Figure 4 shows the same results as Fig. 3 but for SAT. We determined SAT from model-simulated LST and air temperature at the lowest layer of the atmosphere with the help of the stability index (Richardson number). The results of CReSS_CTL_5 km and CReSS OPT $5 \mathrm{~km}$ showed similar horizontal patterns to those of the $\bar{A}$ WS observation data during both day- and nighttime, especially over the central part of the UAE. The difference in the 45-point average SAT between the AWS observations and CReSS simulation results did not change significantly; the difference slightly increased from $0.2 \mathrm{~K}$ in the CTL to $-1.8 \mathrm{~K}$ in the OPT experiment during the daytime and decreased from $1.7 \mathrm{~K}$ in the CTL to $-0.6 \mathrm{~K}$ in the OPT experiment at night. As a result, CReSS_OPT $5 \mathrm{~km}$ reproduced a realistically large difference between LST and SAT during the daytime. Moreover, CReSS OPT $5 \mathrm{~km}$ significantly improved the reproducibility of the surface air relative humidity during both day- and nighttime (Fig. S2). This optimization of land surface parameters mainly improved the partitioning of the net radiation flux into latent and sensible heat fluxes, suppressing excessive latent heat flux and increasing sensible heat flux (Fig. S3). From these results, the performance of CReSS_OPT_5km was improved in terms of simulating both LST (a)

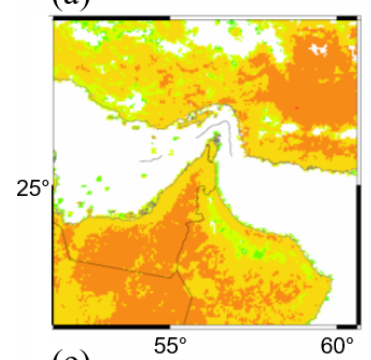

(e)

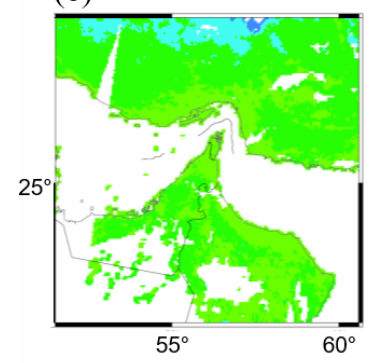

(b)

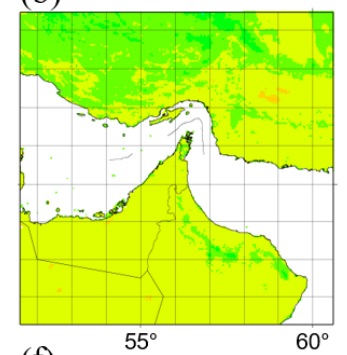

(f)

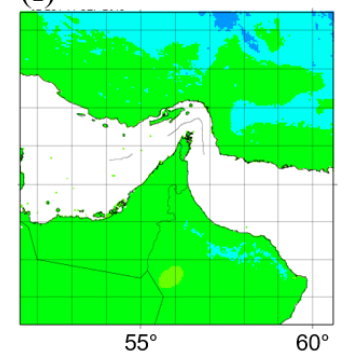

(c)

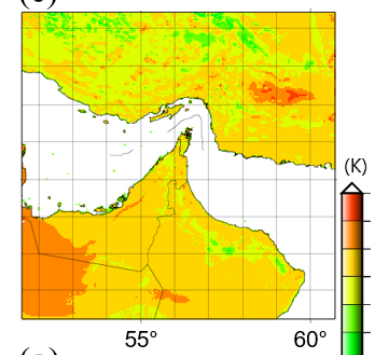

(g)

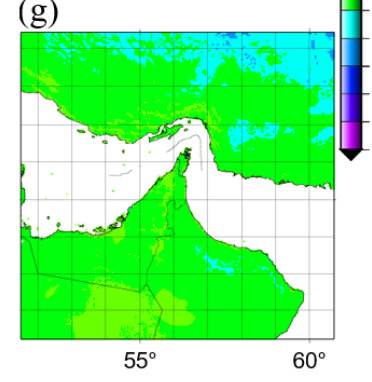

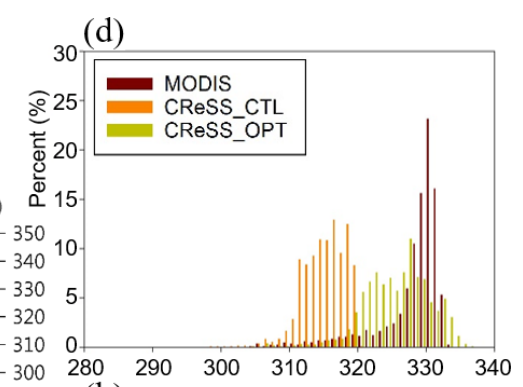

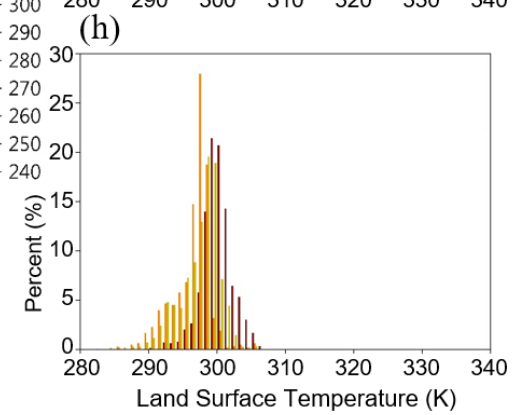

Fig. 3. Horizontal distribution of land surface temperature during daytime by (a) Aqua/MODIS, (b) CReSS_CTL_5km, and (c) CReSS_OPT_5km, and (d) a histogram, at 14:00 LT on 10 September 2015, as well as at night by (e) Aqua/MODIS, (f) CReSS_CTL_5km, and (g) CReSS_OPT_5km, and (h) a histogram, at 02:00 LT on 11 September 2015. MODIS overpass times were at 13:20 and 01:20 LT. The red, orange, and yellow bars in (d) and (h) denote the results of the MODIS observations and the CReSS_CTL and CReSS_OPT experiments. The histograms of land surface temperature at 02:00 LT from CReSS_CTL and CReSS_OPT experiments were calculated excluding areas lacking MODIS data. 
(a)

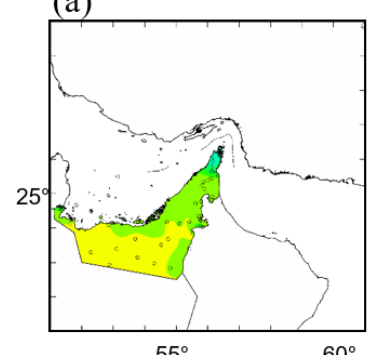

(e)

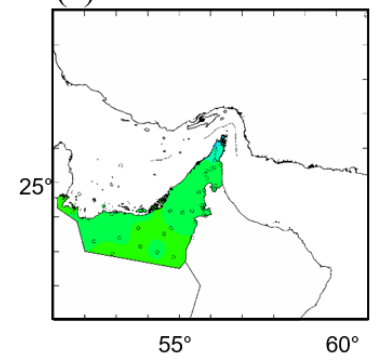

(b)

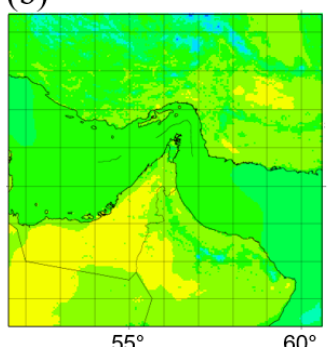

(f)

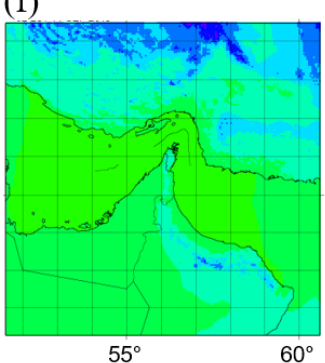

(c)

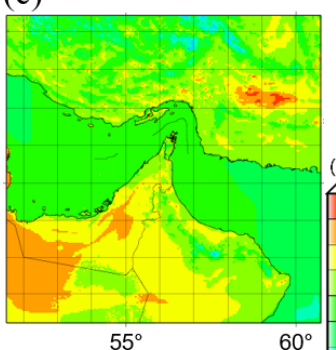

(g)

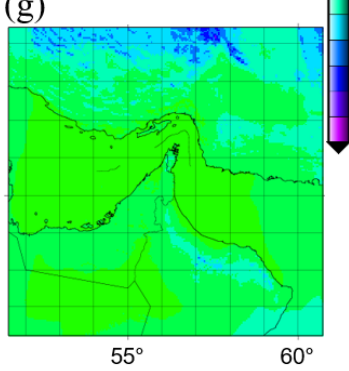

(d)

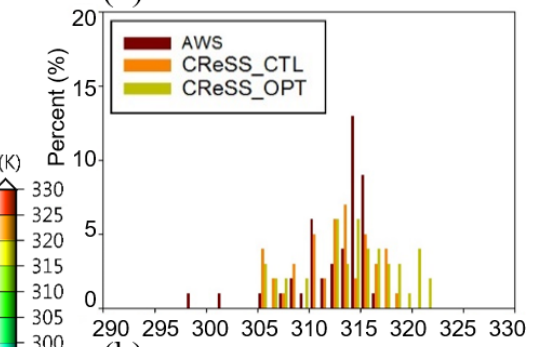

(h)

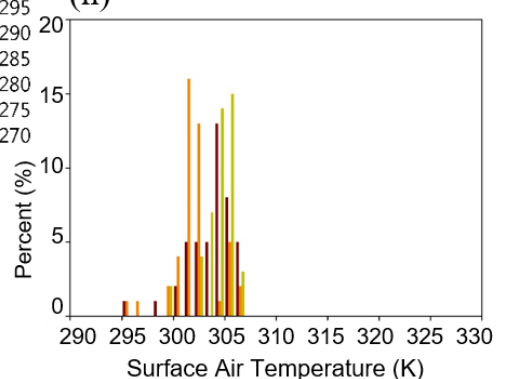

Fig. 4. Horizontal distribution of surface air temperature during daytime by (a) automatic weather station (AWS), (b) CReSS_CTL_5km, and (c) CReSS OPT 5km, and (d) a histogram, at 14:00 LT on 10 September 2015, as well as at night by (e) AWS, (f) CReSS_CTL_5km, and (g) CReSS_OPT_5km, and (h) a histogram, at 02:00 LT on 11 September 2015. AWS data from 45 observation sites were interpolated to create the continuous distribution of SAT over the United Arab Emirates. The red, orange, and yellow bars in (d) and (h) denote the results of the AWS observations and the CReSS_CTL and CReSS_OPT experiments.

and SAT, although CReSS OPT $5 \mathrm{~km}$ still slightly underestimated LST and overestimated SATT during both the day- and nighttime. At the AWS sites in Al Ain and Al Qua'a, the difference between observations and CReSS_OPT_5km significantly decreased for both LST and SAT (Fig. Sī).

The vertical profile of soil temperature at the Al Ain site showed reasonable agreement between the observations and CReSS_OPT_5km simulation results (Fig. S4). The optimized parameters improved not only LST, SAT, and surface air relative humidity but also the vertical profile of soil temperature. Therefore, we conclude that the values of the parameters determined by the optimization experiments are representative of arid and semiarid regions.

By adopting these optimized land surface parameters (except soil temperature at the deepest layer) for the cold and warm seasons, the CReSS OPT $5 \mathrm{~km}$ simulations showed a marked improvement in $\mathrm{LST}^{\text {(from }} 15.9$ to $6.5 \mathrm{~K}$ in the daytime and 4.8 to $4.4 \mathrm{~K}$ at night for the cold season and from 12.9 to $2.2 \mathrm{~K}$ in the daytime and 4.7 to $3.6 \mathrm{~K}$ at night for the warm season), similar to the study period (9-11 September 2015); there was also no significant change in SAT (from 0.6 to $-0.4 \mathrm{~K}$ in the daytime and 0.8 to $-1.0 \mathrm{~K}$ at night for the cold season and from 0.6 to $-1.6 \mathrm{~K}$ in the daytime and 1.1 to $-2.1 \mathrm{~K}$ at night for the warm season). Here, monthly climatological data for the UAE, obtained observationally by Takano et al. (1999), were used for the soil temperature at the deepest layer. Overall, these results indicate that the optimized parameters were valid not only for 9-11 September 2015 but also for numerical simulations over the UAE and surrounding areas throughout the year.

\subsection{Reproducibility of clouds and precipitation}

The CReSS_OPT_5km simulation showed improved reproduction of diurnal convective clouds over desert areas to some extent, but it was still not sufficient for reproducing isolated precipitating diurnal convective clouds. To evaluate the reproducibility of such clouds over the UAE, the CReSS_OPT simulation was re-run at a $1-\mathrm{km}$ horizontal resolution (CReSS_OPT_1 km). The simulation results of CReSS_OPT_1 km show that the deviations in LST from CReSS_OPT $5 \overline{\mathrm{km}}$ were 1 and $0 \mathrm{~K}$ during the dayand nighttime, respectively. This implies that LST is not affected by the horizontal resolution of the model.
Figure 5 shows the temporal variations in the horizontal distributions of column maximum reflectivity from 13:00 to 16:00 LT on 10 September 2015 (obtained from UAE National Center of Meteorology operational radar observation, CReSS_CTL_5km, CReSS_CTL_1km, CReSS_OPT_5km, and CReSS_OPT_1 km). CReSS_OPT_1 km drastically improved the horizontal distributions of the column maximum reflectivity compared with CReSS CTL_5km and CReSS_OPT_5km. Even compared with CReSS $\mathrm{CTL}_{-} 1 \mathrm{~km}$, it showed a substantial improvement in reproducing diurnal convective clouds (15:00 and 16:00 LT), especially over deserts in the UAE and low mountains in northeastern UAE, capturing the characteristics of the radar observations. Note that diurnal convective clouds over the Omani mountain areas were well simulated by the model due to strong influences of the summertime Indian monsoon, high elevations of the mountains, and solar radiation, regardless of the optimization of land surface parameters.

Figure 6 shows the horizontal distributions and histograms of both the cloud fraction (CF) and cloud top temperature (CTT) at 13:00 LT on 10 September 2015. Cloud top properties, such as CTT and CF, derived from Aqua/MODIS data at a $5 \times 51-\mathrm{km}$ pixel resolution were analyzed over the cloudy portion of the $5 \times$ 5 pixel arrays, wherein the cloud pixels identified by the probably cloudy and cloudy bits of the cloud mask were averaged to reduce noise. The average CTT was only calculated for $5 \times 5$ pixel arrays that contained four or more cloudy pixels (Platnick et al. 2015). A similar algorithm was applied to the CReSS simulation results.

Around 13:00 LT on 10 September 2015, diurnal convective clouds just began to form over the low mountains in northeastern $\mathrm{UAE}$ and the desert northwest of Al Ain. A few convective clouds forming over these areas were seen only in CReSS_OPT_1 km simulation results (Figs. 6c and 6g), although 13:00 LT was still too early for the influence of the land surface processes on cloud formation to clearly appear. Then, as shown in Fig. 5, many convective clouds continued to form and develop in these areas and brought precipitation by around 16:00 LT.

The CF within the $5 \times 5$ pixel arrays showed reasonable agreement between the Aqua/MODIS observations and CReSS OPT $1 \mathrm{~km}$ simulation, although the occurrence frequencies of CFs less than 0.1 and greater than 0.8 obtained from the CReSS simulation were over- and underestimated, respectively. The mean 
(a)

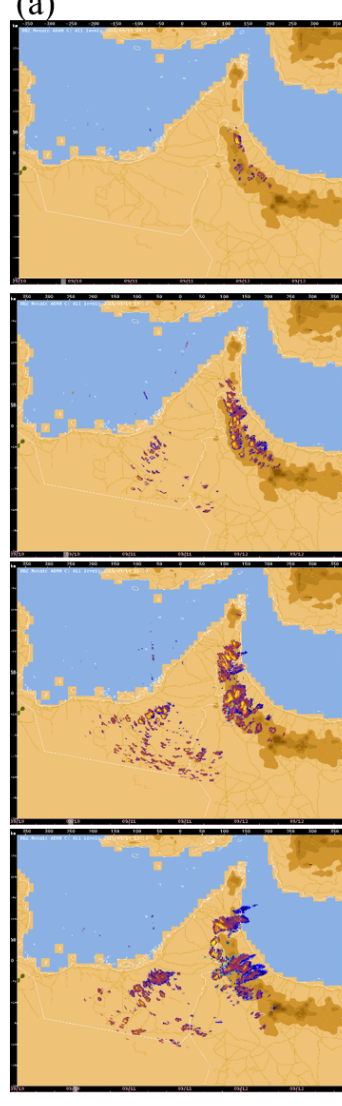

(b)
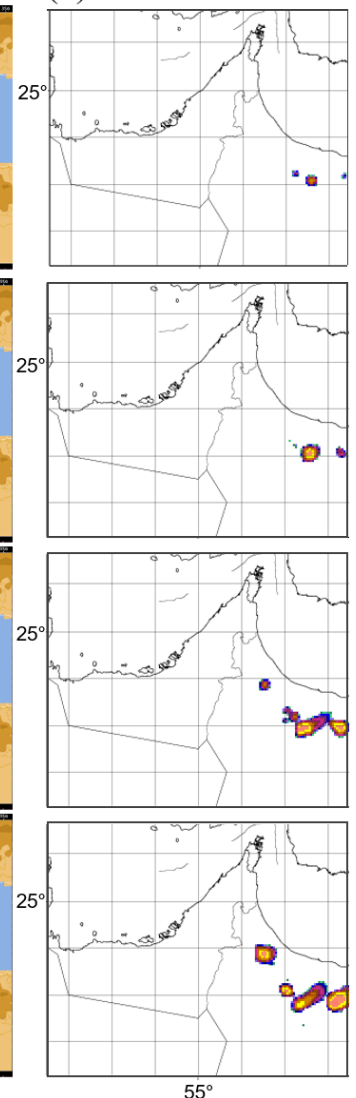

(c)
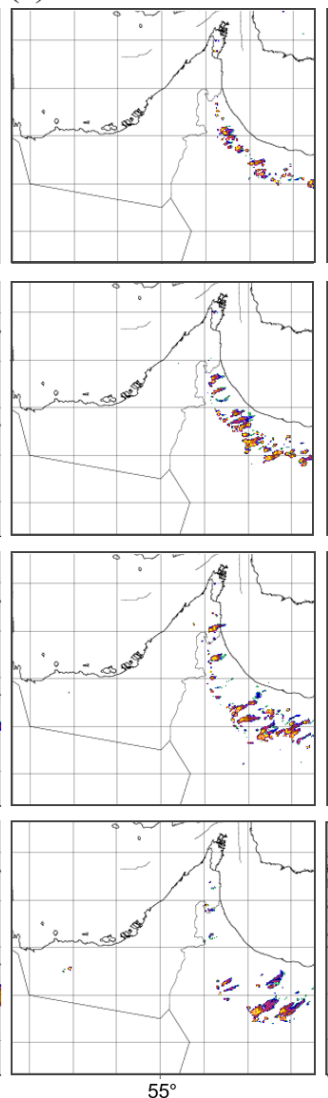

(d)

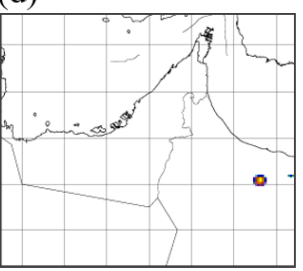

(e)
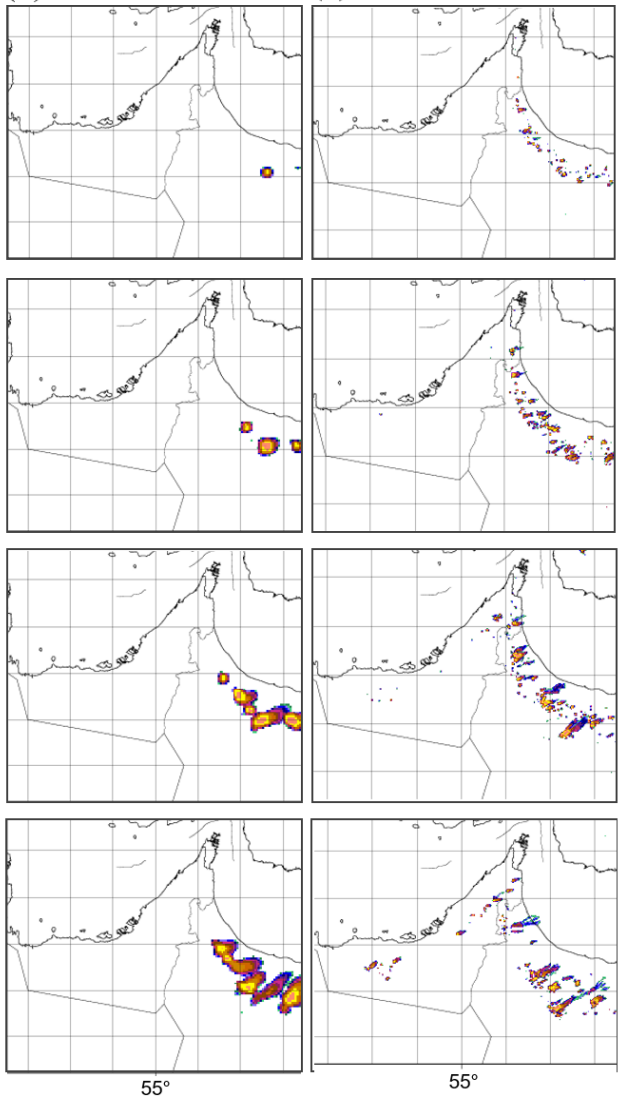

(dBZ)

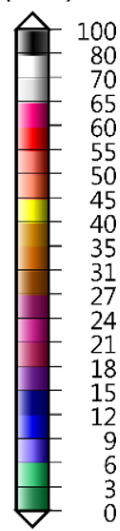

Fig. 5. Horizontal distribution of column maximum reflectivity obtained from (a) radar observations, (b) CReSS CTL 5km, (c) CReSS CTL 1 km, (d) CReSS_OPT_5km, and (e) CReSS_OPT_1 km experiments at 13:00 LT (first row), 14:00 LT (second row), 15:00 LT (third row), and 16:00 LT (fourth row) on 10 September 2015. Panel (a) is from the United Arab Emirates National Center of Meteorology's operational C-band radar composite combining Al Ain and Abu Dhabi radar data. Brown shading indicates the terrain elevations at $500 \mathrm{~m}$ intervals.

(a)

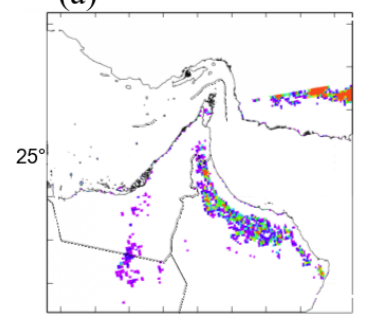

(e)

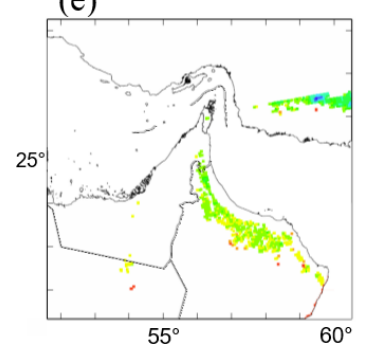

(b)

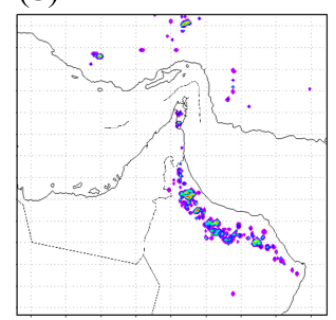

(f)

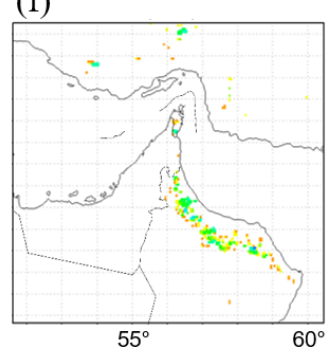

(c)

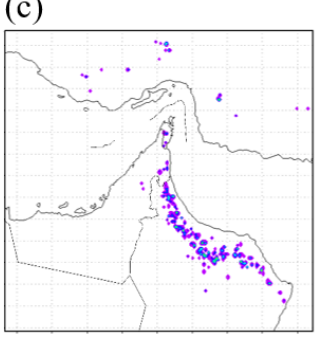

(g)

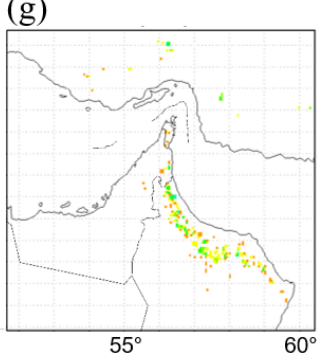

(d)

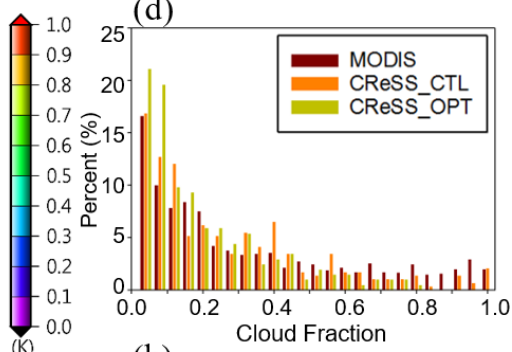

(h)

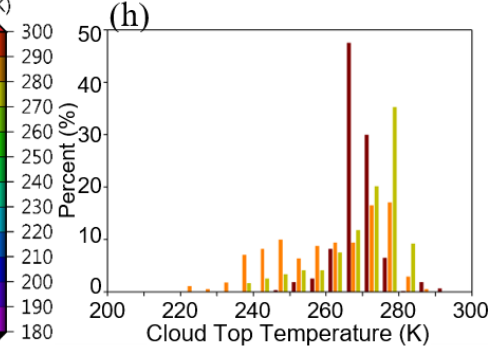

Fig. 6. Horizontal distribution of the cloud fraction by (a) Aqua/MODIS, (b) CReSS_CTL_1km, and (c) CReSS_OPT_1 km, and (d) a histogram, as well as cloud top temperature by (e) Aqua/MODIS, (f) CReSS_CTL_1km, and (g) CReSS_OPT_1km, and (h) a histogram, at 13:00 LT on 10 September 2015. The Aqua/MODIS overpass time was 13:20 LT.

CTT showed good agreement between the Aqua/MODIS observations and CReSS simulation results, although the peak of the CTT histogram from the CReSS model appeared at a temperature several degrees higher than that from the Aqua/MODIS data. Both the Aqua/MODIS observations and CReSS_OPT_1 km simulation results indicated cloudy regions with a CF less than 0.2 and a CTT around freezing point over the mountain and desert areas, which is indicative of the existence of low- and/or mid-level isolated 
convective clouds.

Although the overall CF and CTT simulated by CReSS_OPT $1 \mathrm{~km}$ were slightly under- and overestimated, respectively, the CReSS_OPT_1 km results represented the horizontal distribution of diurnal convective clouds reasonably well over the desert and mountain areas. The differences between the MODIS observations and CReSS results may be attributed to differences in the algorithms used to analyze CTT and CF. There may also have been errors, bias, or uncertainties associated with the cloud-top property algorithm of the MODIS data analysis or a slight inadequacy in the reproducibility of diurnal convective clouds by the CReSS model. The accuracy of diurnal convective cloud tops simulated by the CReSS model will be further investigated using data from in-situ aircraft measurements and/or active remote sensors aboard satellites in future work. Regardless, this study indicates that CReSS OPT $1 \mathrm{~km}$ properly simulates cloud formation and precipitation development over both deserts and mountains. The CReSS model therefore demonstrates acceptable reproducibility of the cloud and precipitation system over the UAE and surrounding areas.

\section{Conclusions}

To conduct forecast or hindcast simulations for rain enhancement research over the UAE, we optimized a set of land surface parameters for desert areas in the CReSS model based on the results of LST and SAT sensitivity experiments of eight parameters and improved the performance of the CReSS model for weather simulations over the UAE and surrounding areas. In the optimized experiment, the difference in LST between Aqua/ MODIS observations and CReSS simulation results decreased from 13.0 to $2.3 \mathrm{~K}$ under daytime conditions, mainly due to decreases in evapotranspiration efficiency and thermal diffusivity of soil, and decreased from 3.8 to $2.0 \mathrm{~K}$ under nighttime conditions, mainly due to a decrease in evapotranspiration efficiency and increase in soil temperature at the deepest layer. In addition, CReSS OPT $5 \mathrm{~km}$ reproduced a realistically large difference between LST and SAT during the daytime, where a decrease in the roughness length for momentum and heat/water vapor contributed to the suppression of increasing SAT. The values of land surface parameters determined in the optimization experiments converged to representative values for arid and semi-arid regions. Numerical simulations with CReSS_OPT_1 km appropriately reproduced the formation of diurnal convective clouds and development of precipitation over deserts in the UAE, low mountains in northeastern UAE, and mountains in Oman, demonstrating an acceptable reproducibility of the cloud and precipitation system over the UAE and surrounding areas using the CReSS model.

\section{Acknowledgements}

This study was supported by the National Center of Meteorology, Abu Dhabi, UAE, under the UAE Research Program for Rain Enhancement Science. The authors thank the editor (Dr. Nishizawa) and two anonymous reviewers for their valuable comments and advice, which helped to significantly improve the manuscript.

Edited by: S. Nishizawa

\section{Supplements}

The supplement includes the model configuration information (Table S1), a comparison of the temporal variations in LST and SAT between the observation data and CReSS simulation results (Fig. S1), the horizontal distribution of surface air relative humidity (Fig. S2), the temporal variation of the land surface energy budget (Fig. S3), and the vertical profile of soil temperature (Fig. S4).

\section{References}

Berge, H. F. M. ten, 1990: Heat and water transfer in bare topsoil and the lower atmosphere. Ph.D. Dissertation, Wageningen University, Pudoc Wageningen, $207 \mathrm{pp}$.

Deardoff, J. W., 1980: Stratocumulus-capped mixed layers derived from a three-dimensional model. Bound.-Layer Meteor, 18, 495-527.

Dudhia, J., D. Gill, K. Manning, W. Wang, C. Bruyere, S. Kelly, and K. Lackey, 2004: PSU/NCAR mesoscale modeling system tutorial class notes and user's guide: MM5 modeling system version 3. NCAR.

Hirata, H., R. Kawamura, M. Kato, and T. Shinoda, 2016: Response of rapidly developing extratropical cyclones to seasurface temperature variations over the western Kuroshio-Oyashio confluence region. J. Geophys. Res. Atmos., 121, 3843-3858.

Kanada, S., S. Tsujino, H. Aiki, M. Yoshioka, Y. Miyazawa, K. Tsuboki, and I. Takayabu, 2017: Impacts of SST patterns on rapid intensification of Typhoon Megi (2010). J. Geophys. Res. Atmos., 122, 13245-13262.

Kondo, J., 1976: Heat balance of the China Sea during the air mass transformation experiment. J. Meteor. Soc. Japan, 54, 382-398.

Lee, K.-O., S. Shimizu, M. Maki, C.-H. You, H. Uyeda, and D.-I. Lee, 2010: Enhancement mechanism of the 30 June 2006 precipitation system observed over the northwestern slope of Mt. Halla, Jeju Island, Korea. Atmos. Res., 97, 343-358.

Li, Z. L., B. H. Tang, H. Wu, H. Ren, G. Yan, Z. Wan, I. F. Trigo, and J. A. Sobrino, 2013: Satellite-derived land surface temperature: current status and perspectives. Remote Sens. Environ., 131, $14-37$.

List, R. J., 1966: Smithsonian Meteorological Tables, Smithsonian Institution Press, $527 \mathrm{pp}$.

Murakami, M., T. L. Clark, and W. D. Hall, 1994: Numerical simulations of convective snow clouds over the Sea of Japan; Two-dimensional simulations of mixed layer development and convective snow cloud formation. J. Meteor. Soc. Japan, 72, $43-62$.

Ohigashi, T., and K. Tsuboki, 2007 : Shift and intensification processes of the Japan-Sea Polar-Airmass Convergence Zone associated with the passage of a mid-tropospheric cold core. $J$. Meteor. Soc. Japan, 85, 633-662.

Platnick, S., M. D. King, K. G. Meyer, G. Wind, N. Amarasinghe, B. Marchant, G. T. Arnold, Z. Zhang, P. A. Hubanks, B. Ridgway, and J. Riedi, 2015: MODIS Cloud Optical Properties: User Guide for the Collection 6 Level-2 MOD06/MYD06 Product and Associated Level-3 Datasets. Goddard Space Flight Center, USA, $141 \mathrm{pp}$.

Shinoda, T., A. Higuchi, K. Tsuboki, T. Hiyama, H. Tanaka, S. Endo, H. Minda, H. Uyeda, and K. Nakamura, 2009: Structure of convective circulation in the atmospheric boundary layer over the northwestern Pacific Ocean under a subtropical high. J. Meteor. Soc. Japan, 87, 979-996.

Takano, Y., T. Fukuhara, and K. Sato, 1999: Considerations of seasonal micro-meteorological characteristic in the United Arab Emirates and evaporation-drying after watering the soil by the "soil thermo-hygrometer" method. J. Japan Soc. Hydrolo. Water Resour., 12, 327-337.

Tsuboki, K., and A. Sakakibara, 2007: Numerical prediction of high-impact weather systems, The Textbook for Seventeenth IHP Training Course in 2007, HyARC, Nagoya University, and UNESCO, $281 \mathrm{pp}$.

Wang, C.-C., S.-Y. Huang, S.-H. Chen, C.-S. Chang, and K. Tsuboki, 2016: Cloud-resolving typhoon rainfall ensemble forecasts for Taiwan with large domain and extended range through timelagged approach. Wea. Forecasting, 31, 151-172.

Warner, T. T., 2009: Desert Meteorology, Cambridge University Press, $620 \mathrm{pp}$.

Yamada, H., B. Geng, H. Uyeda, and K. Tsuboki, 2007: Role of the heated landmass on the evolution and duration of a heavy rain episode over a Meiyu-Baiu frontal zone. J. Meteor. Soc. Japan, 85, 687-709.

Zhang, Q., R. Huang, and H. Tian, 2003: A parameterization scheme of surface turbulent momentum and sensible heat over the Gobi underlying surface. Adv. Atmos. Sci., 20, 111-118.

Manuscript received 10 April 2018, accepted 15 November 2018

SOLA: https://www.jstage.jst.go.jp/browse/sola/ 